\title{
Quark condensate seesaw mechanism for neutrino mass
}

\author{
A. Babič $\odot{ }^{1,2,3}$ S. Kovalenko, ${ }^{4,5}$ M. I. Krivoruchenko, ${ }^{6,7}$ and F. Šimkovic $\circledast^{2,3,8}$ \\ ${ }^{1}$ Faculty of Nuclear Sciences and Physical Engineering, Czech Technical University in Prague, \\ 11519 Prague, Czech Republic \\ ${ }^{2}$ Institute of Experimental and Applied Physics, Czech Technical University in Prague, \\ 11000 Prague, Czech Republic \\ ${ }^{3}$ Bogoliubov Laboratory of Theoretical Physics, \\ Joint Institute for Nuclear Research, 141980 Dubna, Russia \\ ${ }^{4}$ Departamento de Ciencias Físicas, Universidad Andres Bello, Sazie 2212, Piso 7, Santiago, Chile \\ ${ }^{5}$ Centro Científico Tecnológico de Valparaíso, Casilla 110-V, Valparaíso, Chile \\ ${ }^{6}$ Institute for Theoretical and Experimental Physics, NRC "Kurchatov Institute," \\ B. Cheremushkinskaya 25, 117218 Moscow, Russia \\ ${ }^{7}$ National Research Center "Kurchatov Institute," Ploshchad' Akademika Kurchatova 1, \\ 123182 Moscow, Russia \\ ${ }^{8}$ Faculty of Mathematics, Physics and Informatics, Comenius University in Bratislava, \\ 84248 Bratislava, Slovakia
}

(Received 27 November 2019; accepted 30 November 2020; published 6 January 2021)

\begin{abstract}
We study a mechanism of generation of Majorana neutrino mass due to spontaneous breaking of chiral symmetry (SBCS) accompanied by the formation of a quark condensate. The effect of the condensate is transmitted to the neutrino sector via lepton-number violating (LNV) lepton-quark dimension-7 operators known in the literature as an origin of the neutrino-mass-independent mechanism of neutrinoless double-beta $(0 \nu \beta \beta)$ decay. The smallness of neutrino masses is due to a large ratio between the LNV scale and the scale of the SBCS. This is a new realization of the seesaw mechanism, which we dub the quark condensate seesaw (QCSS). We examine the predictions of the QCSS for $0 \nu \beta \beta$-decay and neutrino mass spectrum. We will show that our model predicts the normal neutrino mass ordering and narrow ranges of the neutrino masses.
\end{abstract}

DOI: 10.1103/PhysRevD.103.015007

\section{INTRODUCTION}

The smallness of neutrino masses, in comparison with the other Standard Model (SM) fermions, remains a mystery of particle physics theory. A common wisdom suggests that this smallness is related to some broken symmetry. One of the most natural candidates is $\mathrm{U}(1)_{L}$ symmetry of the lepton (L) number, broken at some highenergy scale $\Lambda$. Then at the electroweak scale there appears the $\Delta L=2$ Weinberg operator

$$
\mathcal{O}_{W}=\frac{f}{\Lambda} \overline{L^{C}} H L H
$$

which, after the electroweak symmetry breaking (EWSB) $\sqrt{2}\left\langle H^{0}\right\rangle=v=246 \mathrm{GeV}$, leads to the Majorana neutrino mass

Published by the American Physical Society under the terms of the Creative Commons Attribution 4.0 International license. Further distribution of this work must maintain attribution to the author(s) and the published article's title, journal citation, and DOI. Funded by SCOAP.

$$
m_{\nu}=-f v \frac{v}{\Lambda}
$$

For a generic case with $f \sim 1$ and for $m_{\nu}$ at a sub-eV scale one estimates $\Lambda \sim 10^{14-15} \mathrm{GeV}$, putting lepton number violating (LNV) physics far beyond the experimental reach. This happens in the tree-level realizations of the Weinberg operator (1) in the celebrated seesaw type I, II, and III, where $\Lambda$ is equal to the masses $M$ of the corresponding seesaw messengers which, being very heavy, have no phenomenological significance. In order to escape this situation and open up the possibility for a nontrivial phenomenology, various models have been proposed in the literature (for a recent review see, e.g., Ref. [1]) relaxing the above-mentioned limitation on the LNV scale $\Lambda$. Introducing new symmetries (softly broken), one can forbid the operator (1) at the tree level, while allowing it at certain loop level $l$, so that in (2) there appears a loop suppression factor $f \sim\left(1 / 16 \pi^{2}\right)^{l}$. With the appropriate $l$ the LNV scale $\Lambda$ can be reduced down to phenomenologically interesting values in the $\mathrm{TeV}$ ballpark (see, e.g., Refs. [2-8] and references therein). Another possibility is to resort to symmetries forbidding (1) at all, but allowing higher 
dimension- $(5+n)$ operators which after EWSB provide an extra suppression factor $(v / \Lambda)^{n}$. As in the loop-based models, here, for sufficiently large $n$, the LNV scale $\Lambda$ can be made as low as the current experimental limits. In some models both loop and higher-dimension suppressions can be combined.

In the present paper we consider another class of the SM gauge-invariant effective operators

$$
\mathcal{O}_{7}^{u, d}=\frac{g_{\alpha \beta}^{u, d}}{\Lambda^{3}} \overline{L_{\alpha}^{C}} L_{\beta} H\left\{\left(\bar{Q} u_{R}\right),\left(\overline{d_{R}} Q\right)\right\} .
$$

Here, all the possible $\mathrm{SU}(2)_{L}$ contractions are assumed. The operators (3) were previously studied in the literature as a source of $\Delta L=2$ interactions able to induce $0 \nu \beta \beta$ decay with no explicit dependence on the Majorana neutrino mass [9-12]. On the other hand, it was observed in Ref. [13] that this operator contributes to the Majorananeutrino mass matrix due to spontaneous breaking of chiral symmetry (SBCS) via the light-quark condensate $\langle\bar{q} q\rangle=$ $-\omega^{3} \neq 0$. The latter sets the SBCS scale, so that after the EWSB and SBCS one arrives at the contribution to the Majorana mass matrix of active neutrinos

$$
m_{\alpha \beta}^{\nu}=-\frac{g_{\alpha \beta}}{\sqrt{2}} v \frac{\langle\bar{q} q\rangle}{\Lambda^{3}}=\frac{g_{\alpha \beta}}{\sqrt{2}} v\left(\frac{\omega}{\Lambda}\right)^{3},
$$

with $\quad g_{\alpha \beta}=g_{\alpha \beta}^{u}+g_{\alpha \beta}^{d}$, where $\langle\bar{q} q\rangle \equiv\langle\bar{u} u\rangle \approx\langle\bar{d} d\rangle \approx$ $2\left\langle\overline{u_{L}} u_{R}\right\rangle \approx 2\left\langle\overline{d_{R}} d_{L}\right\rangle$. This is a kind of seesaw formula relating the smallness of the Majorana masses of neutrino with the large ratio between the scale $\Lambda$ of lepton-number violation (LNV) and the scale of chiral-symmetry breaking $\omega=-\langle\bar{q} q\rangle^{1 / 3}$. We call the relation (4) quark condensate seesaw (QCSS) formula. Taking

$$
\langle\bar{q} q\rangle^{1 / 3} \approx-283 \mathrm{MeV}
$$

from a renormalized lattice $\mathrm{QCD}$ within the $\overline{\mathrm{MS}}$ scheme at a fixed scale $\mu=2 \mathrm{GeV}$ [14] and $\Lambda \sim$ a few $\mathrm{TeV}$ we get the neutrino mass in the sub-eV ballpark.

In the next section we study implications of the requirement of the dominance of the operator (3) for ultraviolet (UV) model building and certain phenomenological aspects of the QCSS. In Sec. III we extract limits on the couplings of nonstandard neutrino-quark contact interactions appearing in QCSS. Then, we analyze contributions of the operators (3) to neutrinoless double-beta $(0 \nu \beta \beta)$ decay and derive strong limitations on the QCSS mechanism from this LNV process.

\section{DOMINANCE OF QCSS AND LIGHT-QUARK MASSES}

Here we discuss the conditions for the dominance of the operator (3) in the Majorana neutrino mass matrix. As usual, this can be guaranteed by imposing on the theory an appropriate symmetry group $\mathcal{G}$ which could be either continuous or discrete. General properties of this kind of symmetries were studied in Ref. [13]. This symmetry must forbid the Weinberg operator (1), but allow the operator $\mathcal{O}_{7}^{q}$ in Eq. (3). Therefore, the lepton bilinear $L L$ must be a $\mathcal{G}$ nonsinglet. Requiring that $\mathcal{G}$ remains a good symmetry after the EWSB and still forbids any contribution to the Majorana-neutrino mass term

$$
\mathcal{L}_{\mathrm{M}}=-\frac{1}{2} \sum_{\alpha \beta} \overline{\nu_{\alpha L}^{C}} m_{\alpha \beta}^{\nu} \nu_{\beta L}+\text { H.c. }
$$

while allowing the quark-lepton coupling

$$
\mathcal{L}_{7}=\frac{1}{\sqrt{2}} \sum_{\alpha \beta} \frac{v}{\Lambda^{3}} \overline{\nu_{\alpha L}^{C}} \nu_{\beta L}\left(g_{\alpha \beta}^{u} \overline{u_{L}} u_{R}+g_{\alpha \beta}^{d} \overline{d_{R}} d_{L}\right)+\text { H.c. }
$$

implies that we claim the SM Higgs $H$ to be a $\mathcal{G}$-singlet. Thus, the condition of $\mathcal{G}$ invariance of the operator (3) requires that one of the quark bilinears $\left(\bar{Q} u_{R}\right)$ and $\left(\overline{d_{R}} Q\right)$ or both be $\mathcal{G}$ nonsinglets. The latter implies that the Yukawa couplings of $u$ and $d$ quarks

$$
H^{\dagger} \bar{Q} u_{R}, \quad H \bar{Q} d_{R}
$$

are not $\mathcal{G}$-invariant and forbidden by this symmetry. Therefore, the light quarks do not receive their masses as a result of the EWSB. In principle, this is in line with the fact that the light quarks $u, d$ are particular among other quarks by being much lighter than the others. However, the statement of vanishing masses $m_{u, d}=0$, or even one of them, seems to contradict the known results of lattice calculations [15] and experimental data on the light meson masses. Therefore, small $m_{u, d} \neq 0$ must be generated in some way to make our scenario viable. In principle, for this scenario it is not necessary that both Yukawa couplings in Eq. (8) are forbidden. As seen from (3), it is sufficient that only one of them, say the $u$-quark Yukawa coupling, be forbidden as suggested in Ref. [13].

Thus, we assume that $m_{d}$ is generated via an effective Yukawa coupling (8), realized at some loop level for making it sufficiently small in comparison to the other heavier quarks. On the other hand, we require that the Yukawa coupling for u-quark be forbidden by the symmetry $\mathcal{G}$ so that above the electroweak scale the current u-quark mass is $m_{u}=0$. Therefore, in this setup we require that the quark bilinears transform under the $\mathcal{G}$-symmetry group as

$$
\mathcal{G} \text { - nonsinglet: }\left(\bar{Q} u_{R}\right), \quad \mathcal{G} \text { - singlet: }\left(\overline{d_{R}} Q\right) .
$$

Consequently, we should set $g^{d}=0$ in Eq. (3). Note that the case of the vanishing current $u$-quark mass has long been considered as one of the possible solutions of the strong $C P$ 
problem, allowing us to rotate away the $C P$-violating angle $\theta$ from the QCD Lagrangian.

However, the key question here is whether this is compatible with the lattice value $m_{u}^{\text {latt }}=2.78 \pm 0.19 \mathrm{MeV}$ [15] and the light meson masses. We start with the observation that $m_{u}=0$ at some high-energy cutoff scale does not prevent generation of a nonzero effective quark mass $m_{u}^{\text {eff }}$ at low sub-GeV scales. Here there are several sources of $m_{u}^{\text {eff }} \neq 0$ rooting in the strong interaction dynamics.

First, we note that in a generic effective theory the light quark masses can be generated due to chiral symmetry breaking via the effective SM-invariant operators

$\mathcal{O}_{6}^{q q}=\frac{\kappa^{q q}}{\Lambda_{q q}^{2}} \bar{Q} Q_{R} \overline{Q_{R}} Q, \quad \mathcal{O}_{6}^{u d}=\frac{\kappa^{u d}}{\Lambda_{u d}^{2}} \bar{Q} u_{R} \bar{Q} d_{R}$,

with $Q_{R}^{T}=(u, d)_{R}$ being right-handed isodoublet. Here, $\Lambda_{q q}, \Lambda_{u d}$ are scales of the physics underlying these operators. Both operators in Eq. (10) among their components have

$$
\begin{aligned}
\mathcal{O}_{6}^{q q} & \sim \overline{u_{L}} u_{R} \overline{u_{R}} u_{L}+\overline{d_{L}} d_{R} \overline{d_{R}} d_{L}, \\
\mathcal{O}_{6}^{u d} & \sim \overline{u_{L}} u_{R} \overline{d_{L}} d_{R},
\end{aligned}
$$

which can contribute to the effective light-quark mass $m_{u, d}^{\text {eff }}$ after spontaneous breaking of chiral symmetry and formation of the quark condensate $\langle\bar{q} q\rangle$. We note that the operator $\mathcal{O}_{6}^{q q}$ conserves chiral symmetry while $\mathcal{O}_{6}^{u d}$ breaks it explicitly. In our setup (9) the operator $\mathcal{O}_{6}^{u d}$ is forbidden by $\mathcal{G}$-symmetry. In a scenario with $m_{u}=m_{d}=0$ above the electroweak scale this operator is allowed and can have interesting implications, if its scale $\Lambda_{u d}$ is not very high. This scenario will be addressed elsewhere.

The chiral symmetric operator $\mathcal{O}_{6}^{q q}$ in Eq. (10) is well known in the context of the Nambu-Jona-Lasinio model considered as chiral low-energy effective theory of QCD. Recall, that in this approach the one-gluon exchange diagram with the amplitude

$$
\left(\bar{Q} \gamma^{\mu} \lambda^{a} Q\right) D_{\mu \nu}^{(G) a b}\left(\overline{Q_{R}} \gamma^{\nu} \lambda^{b} Q_{R}\right)
$$

turns to a pointlike 4-quark operator in a truncated theory, where the gluon propagator $D^{(G)}\left(k^{2}\right)$ is replaced with $g^{\mu \nu} / \Lambda_{\mathrm{QCD}}^{2}$. Here, $\Lambda_{\mathrm{QCD}} \sim 100 \mathrm{MeV}$ is a characteristic scale of nonperturbative QCD. After Fierz rearrangement in (12) one finds the operator $\mathcal{O}_{6}^{q q}$ in Eq. (10) with the scale $\kappa^{q q} / \Lambda_{q q}^{2} \sim-\alpha_{s} /\left(4 \Lambda_{\mathrm{QCD}}^{2}\right)$. After spontaneous breaking of chiral symmetry this operator renders a contribution

$$
m_{u, d}^{\mathrm{eff}}=m_{u, d}^{C}=\kappa \frac{\langle\bar{q} q\rangle}{\Lambda_{q q}^{2}}=\frac{\alpha_{s}}{4} \omega\left(\frac{\omega}{\Lambda_{\mathrm{QCD}}}\right)^{2} \sim \omega
$$

to the masses of $u$ and $d$ quarks, converting them to the so-called constituents quarks with an effective mass $m_{q}^{C} \sim 100 \mathrm{MeV}$.

However, spontaneous breaking of chiral symmetry cannot be the only source of the quark masses. They must also have a piece $m_{u, d} \neq 0$, which breaks chiral symmetry explicitly. According to the Gell-Mann-Oakes-Renner relation (B8), this is needed in order to pions, as Goldstone bosons of the spontaneous breaking of chiral symmetry, acquire nonzero masses. In our setup (9) $d$-quark has $m_{d} \neq 0$ at a high-energy cutoff scale due to Yukawa coupling (8) explicitly breaking chiral symmetry. However, $u$-quark is also required to contribute to this explicit breaking as follows from the analysis of the meson mass spectrum (see, for instance Ref. [16] and references therein). Here we adopted $m_{u}=0$ above the electroweak scale. In Ref. [17] it was advocated that the next to leading order chiral Lagrangian terms together with the QCD instanton are able to induce at the QCD scale $\Lambda_{\mathrm{QCD}} \sim$ $100 \mathrm{MeV}$ a contribution to $u$-quark mass explicitly breaking chiral symmetry. The resulting effective mass is compatible with the lattice result [15]. The value of the $u$-quark effective mass due to these two was estimated in Ref. [16] with the result

$$
m_{u}^{C h S B}=2.33 \pm 0.20 \mathrm{MeV} .
$$

According to [16] this value is compatible with the light meson masses. However, there is certain tension with the lattice value $m_{u}^{\text {latt }}=2.78 \pm 0.19 \mathrm{MeV}$ [15]. In our opinion this situation requires further study and clarification. Having this point in mind we adopt in this paper the setup (9) and examine its phenomenological consequences.

Before this, the following important comment is in order. In our scenario it is crucial that a nonzero effective mass of $u$-quark, $m_{u}^{\text {eff }}$, is generated at a low scale of the order of the typical QCD scale $\Lambda_{\mathrm{QCD}} \sim 100 \mathrm{MeV}$. In fact, if $u$-quark acquires a mass $m_{u}^{\text {eff }} \neq 0$, regardless of its origin, one can close the $\bar{Q} u_{R}$ legs of the operator $\mathcal{O}_{7}^{u}$ in Eq. (3) via the $u$-quark mass term [18] $m_{u}^{\text {eff }}$ as shown in Fig. 1. This will lead to a 1-loop contribution to the Majorana neutrino mass, which can be estimated as

$$
m_{\nu}^{1-\text { loop }} \sim \frac{1}{\sqrt{2}} \frac{g_{\alpha \beta}^{u}}{4 \pi^{2}} v \frac{1}{\Lambda^{3}} m_{u}^{\text {eff }} \Lambda_{u}^{2},
$$

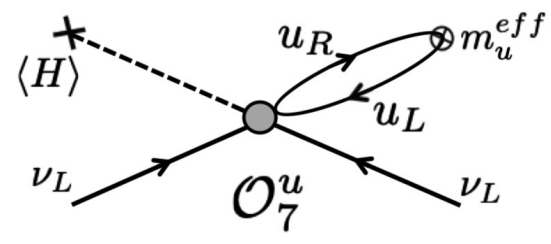

FIG. 1. The loop contribution, $m_{\nu}^{1-\text { loop }}$, of the operator $\mathcal{O}_{7}^{u}$ to the neutrino mass. 
where, $\Lambda_{u}$ is a scale at which $m_{u}^{\text {eff }}$ is generated. In our model $m_{u}^{\text {eff }}=m_{u}^{C}+m_{u}^{C h S B}$, where $m_{u}^{C}$ and $m_{u}^{C h S B}$ are given in (13) and (14), respectively. Both these contributions are generated at a scale around $\Lambda_{\mathrm{QCD}} \sim 100 \mathrm{MeV}$. At a higher scales they are rapidly decreasing, as any nonperturbative QCD effect, providing cutoff in the loop integral in Fig. 1. Thus, we substitute $\Lambda_{u}=\Lambda_{\mathrm{QCD}}, m_{u}^{\mathrm{eff}}=m_{u}^{C}+m_{u}^{C h S B} \approx m_{u}^{C}$ in (15) and have the following neutrino mass with the considered 1-loop correction

$$
m_{\alpha \beta}^{\nu} \simeq \frac{g_{\alpha \beta}}{\sqrt{2}} v\left(\frac{\omega}{\Lambda}\right)^{3}\left(1+\frac{\alpha_{s}}{16 \pi^{2}}\right) .
$$

The 1-loop correction is small and irrelevant for our estimations based on Eq. (4) with $g_{\alpha \beta}=g_{\alpha \beta}^{u}$. Let us recall that in our setup (9) we have $g_{\alpha \beta}^{d}=0$. It is worth noting that in general the loop in Fig. 1 could present a problem. In the case, if $\Lambda_{u} \sim \Lambda \sim 1 \mathrm{TeV}$ its contribution to neutrino mass would be unacceptably large $m_{\nu} \sim 10^{3}-10^{5} \mathrm{eV}$. As we have shown our setup is free of this problem.

\section{LIMITS ON LNV LEPTON-QUARK INTERACTIONS}

Let us examine phenomenological limits on the strength of the effective LNV lepton-quark interactions predicted by the QCSS. These interactions are derived from (3) and (7). It is convenient to introduce the dimensionless parameters

$$
\varepsilon_{\alpha \beta}=\frac{g_{\alpha \beta} v / \Lambda^{3}}{G_{\mathrm{F}}},
$$

giving a measure of the relative strength of the four-fermion interactions (7) with respect to the Fermi constant $G_{\mathrm{F}} \approx$ $1.166 \times 10^{-5} \mathrm{GeV}^{-2}$ of the standard weak interactions. We denoted $g_{\alpha \beta} \equiv g_{\alpha \beta}^{u}$. The latter was introduced in Eq. (3). As discussed in the previous section, in our setup (9) we set $g_{\alpha \beta}^{d}=0$.

Here, assuming the dominance of the QCSS in the Majorana mass of neutrinos, we can extract limits on $\varepsilon_{\alpha \beta}$ from the neutrino-oscillation data since, according to Eq. (4), they are directly related to the elements of the neutrino mass matrix

$$
\varepsilon_{\alpha \beta}=\frac{g_{\alpha \beta} v / \Lambda^{3}}{G_{\mathrm{F}}}=-\frac{m_{\alpha \beta}^{\nu} /\langle\bar{q} q\rangle}{G_{\mathrm{F}} / \sqrt{2}} .
$$

Using Eqs. (A5)-(A10), we relate these LNV lepton-quark parameters to the neutrino-oscillation parameters. The current values of the latter we take from Ref. [19]. Then, varying the $C P$ phases in the intervals $\delta \in[0,2 \pi)$ and $\alpha_{1}, \alpha_{2} \in[0, \pi)$, we find the exclusion plots in the plane $\left(m_{0},\left|\varepsilon_{\alpha \beta}\right|\right)$ shown in Fig. 2 for the best-fit values of the neutrino oscillation parameters $\theta_{i j}$ and $\Delta m_{i j}^{2}(i, j=1,2,3)$.

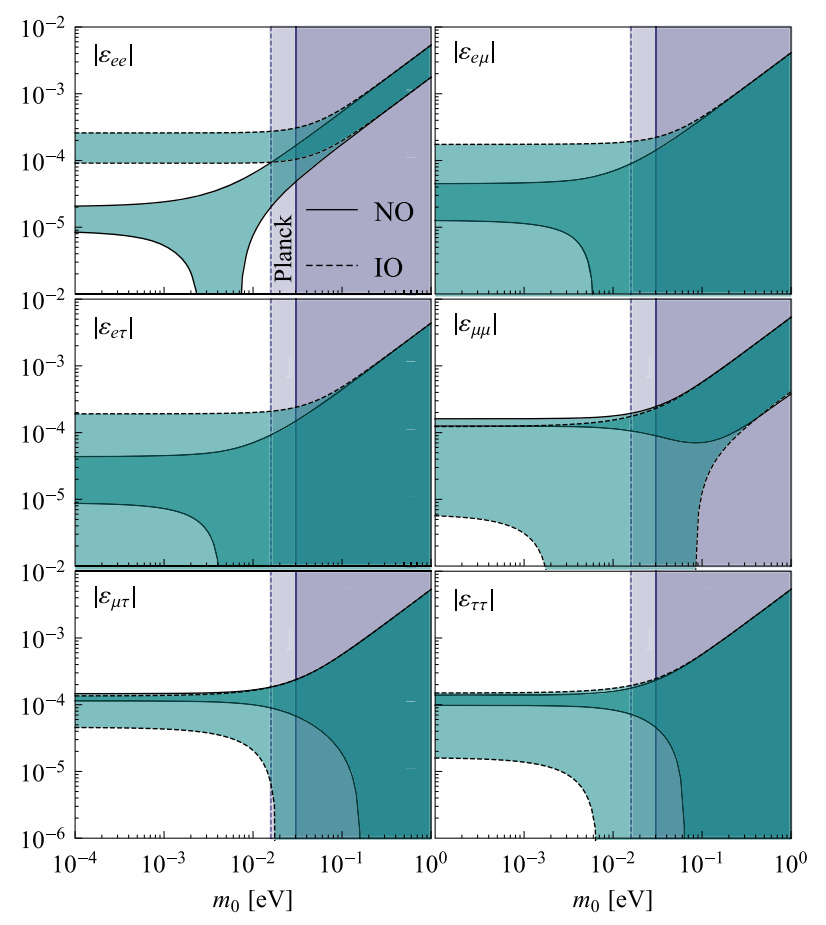

FIG. 2. Predictions of QCSS for the nonstandard neutrinoquark couplings $\left|\varepsilon_{\alpha \beta}\right|$ defined in Eq. (18) vs the lightest-neutrino mass $m_{0}$. The allowed gray regions between the curved lines are derived using current best-fit values of the neutrino-oscillation parameters [19] and $C P$ phases $\delta \in[0,2 \pi)$ and $\alpha_{1}, \alpha_{2} \in[0, \pi)$. The solid and dashed lines refer to the normal (NO) and inverted (IO) orderings of the neutrino masses, respectively. The vertical gray bands represent the regions excluded by Planck measurements at $95 \%$ C.L. [20]. The horizontal gray bands in the first plot correspond to the regions excluded by KamLAND-Zen 90\% C.L. limits on $0 \nu \beta \beta$-decay [21]. The solid and dashed horizontal limiting lines refer to the scenarios with and without the nuclearmatter effect discussed in Sec. IV, respectively.

As is known, the upper cosmological limit on the sum of neutrino masses

$$
\sum_{i} m_{i}<0.12 \mathrm{eV} \text { at 95\%C.L. }
$$

set by the Planck measurements [20,22], impose the limit on the mass $m_{0}$ of the lightest neutrino. Applying Eqs. (A3)-(A4), one finds

$$
m_{0}<30.1 \mathrm{meV}, \quad 15.9 \mathrm{meV}
$$

for the normal (NO) and inverted (IO) neutrino-mass orderings, respectively. These limits are shown in Fig. 2 as vertical bands.

It is instructive to show allowed ranges $\left(\varepsilon_{\alpha \beta}^{\min } ; \varepsilon_{\alpha \beta}^{\max }\right)$ of the LNV lepton-quark interaction parameters $\varepsilon_{\alpha \beta}$ from (18). These ranges extracted from the exclusion plots in Fig. 2 for $\mathrm{NO}$ and $\mathrm{IO}$ are 


$$
\begin{aligned}
& \left|\varepsilon_{\alpha \beta}^{\mathrm{NH}}\right|=\left(\begin{array}{ccc}
(0 ; 1.7) & (0 ; 1.3) & (0 ; 1.5) \\
& (0.9 ; 2.4) & (0.7 ; 2.4) \\
& & (0.5 ; 2.3)
\end{array}\right) \times 10^{-4},
\end{aligned}
$$

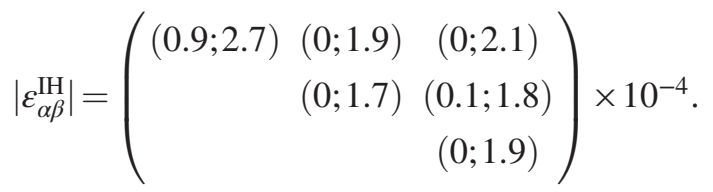

To the best of our knowledge, the only analysis of phenomenological limits on the lepton-quark interaction strength $\varepsilon$ from Eq. (17) existing in the literature is given in Refs. [13,23] where the SN 1987A and meson decays were studied. In the former case these limits are in the range $\varepsilon<10^{-3}$, which is an order of magnitude weaker than our limits in Eqs. (21)-(22). As to the LFV meson decays, reasonable limits on $\varepsilon$-parameters cannot be extracted from the experimental data. Indeed, considering as an example the LNV decay $K^{+} \rightarrow \pi^{-} \mu^{+} \mu^{+}$, one finds [24] for its branching ratio $\operatorname{Br}\left(K^{+} \rightarrow \pi^{-} \mu^{+} \mu^{+}\right) \sim\left|\varepsilon_{\mu \mu}\right|^{2} \times 10^{-30}$, which should be compared with the current experimental upper bound $\operatorname{Br}\left(K^{+} \rightarrow \pi^{-} \mu^{+} \mu^{+}\right) \leq 10^{-11}$. Of course, this gives no practical information on the $\varepsilon_{\mu \mu}$-parameter.

\section{QUARK CONDENSATE SEESAW IN NEUTRINOLESS DOUBLE-BETA DECAY}

Let us consider the contribution of the operator $\mathcal{O}_{7}^{u}$ in Eq. (3) to $0 \nu \beta \beta$ decay. After the EWSB this operator generates the following interactions relevant to $0 \nu \beta \beta$-decay

$$
\mathcal{L}_{7}=\frac{G_{\mathrm{F}}}{\sqrt{2}} \varepsilon_{e e}\left(\overline{e_{L}} \nu_{L}^{C} \overline{u_{R}} d_{L}+\overline{\nu_{L}^{C}} \nu_{L} \overline{u_{R}} u_{L}\right)+\text { H.c. },
$$

where $\varepsilon_{e e}$ is defined in Eq. (17). Let us examine the contribution of the effective $\Delta L=2$ interaction terms (23) to $0 \nu \beta \beta$-decay. The first term (23) combined with the SM weak charged-current interaction leads to the contribution shown in Fig. 3(b), which is independent of neutrino mass in the propagator due to chiralities in the vertices

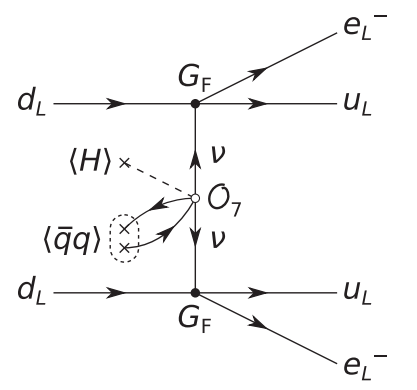

(a)

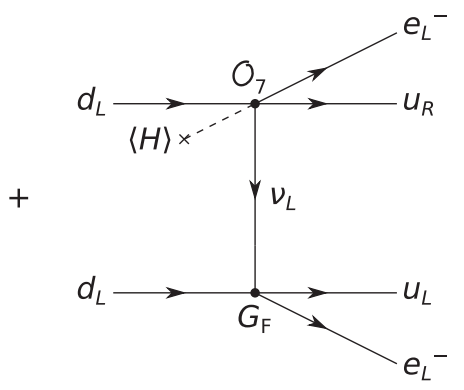

(b)
FIG. 3. Contributions of the effective operators (3) to the $0 \nu \beta \beta$ decay.
$P_{L}\left(m_{\nu}+\not q\right) P_{R}=\not q$. This is a manifestation of the fact that the $\Delta L=2$ is not provided by $m_{\nu}$, but solely by the upper vertex in Fig. 3(b). In our QCSS model the second term in Eq. (23) also contributes to the $0 \nu \beta \beta$-decay via the neutrino-mass mechanism shown in Fig. 3(a). This happens due to the chiral symmetry breaking and formation of the quark condensate. As we discussed in the previous sections, this term is the only source of the neutrino mass in the present model. However, there is a subtlety with the diagram in Fig. 3(a). It describes a process taking place in the nuclear environment, where the chiral quark condensate $\langle\bar{q} q\rangle_{N}$ is suppressed with respect to the one in the vacuum $\langle\bar{q} q\rangle$. As briefly discussed in Appendix B, this suppression is estimated to be a factor-two effect (B9). In what follows, we take this fact into account.

The inverse $0 \nu \beta \beta$-decay half-life in the QCSS model reads (see Appendix C)

$$
\begin{aligned}
\left(T_{1 / 2}^{0 \nu}\right)^{-1} & =\left.\left|\varepsilon_{e e}+\frac{m_{\beta \beta}}{m_{e}} f_{\mathrm{nme}}\right|\right|^{2} g_{A}^{2}\left|M^{\epsilon}\right|^{2} G^{0 \nu} \\
& =\left|\varepsilon_{e e}\right|^{2}\left|1+a_{\nu} f_{\mathrm{nme}}\right|^{2} g_{A}^{2}\left|M^{\epsilon}\right|^{2} G^{0 \nu}
\end{aligned}
$$

where $m_{e}$ is the mass of electron. The standard kinematical phase-space factor $G^{0 \nu}$ is given in Table I and the nuclear structure factor $f_{\text {nme }}$ is given by

$$
f_{\text {nme }}=\frac{g_{A} M^{\nu}}{M^{\varepsilon}} .
$$

Nuclear matrix elements $M^{\nu}$ and $M^{\varepsilon}$ associated with mechanisms in Figs. 3(a) and 3(b), respectively, are presented in Appendix C. $g_{A} \simeq 1.27$ is the axial-vector weak nucleon coupling constant.

In the second row of Eq. (24) we used the prediction of the QCSS model

$$
m_{\beta \beta}=a_{\nu} m_{e} \varepsilon_{e e}, \quad a_{\nu}=\frac{\langle\bar{q} q\rangle_{N}}{\sqrt{2} m_{e}} G_{\mathrm{F}}=-1.83 \times 10^{-4} .
$$

Here, $\langle\bar{q} q\rangle_{N}$ is the value of quark condensate in the nuclear environment of a decaying nucleus, which is different from the vacuum value $\langle\bar{q} q\rangle$ as displayed in Eq. (B9). Consequently, in the QCSS we have

$$
m_{\beta \beta}=\frac{\langle\bar{q} q\rangle_{N}}{\langle\bar{q} q\rangle} m_{e e}^{\nu} \approx 0.5 m_{e e}^{\nu}
$$

where $m_{e e}^{\nu}$ is given by (4) with the chiral condensate in the vacuum. This result contrasts with the conventional neutrino mass models, where $m_{\beta \beta}=m_{e e}^{\nu}$ in the diagonal charged-lepton basis.

With the values of NME for ${ }^{136} \mathrm{Xe}$ given in Appendix C, quark condensate in matter (B9) with (5) and other known parameters we find 
TABLE I. Nuclear matrix elements $M^{\varepsilon}$ (column 3), $M^{\nu}$ (column 4) and their ratio $f_{\text {nme }}=g_{A} M^{\nu} / M^{\epsilon}$ (column 5) calculated within the quasiparticle random-phase approximation (QRPA) method with partial restoration of the isospin symmetry and Argonne V18 nucleonnucleon potential [25]. $M^{\nu}$ and $f_{\text {nme }}$ are given for the unquenched value of the axial-vector coupling constant $g_{A}=1.27$ In columns 6 and 7 the lower experimental limit on the $0 \nu \beta \beta$-decay half-life and the upper constraint on the $\varepsilon_{e e}$ are presented, respectively. The phasespace factors $G^{0 \nu}$ (column 2) are taken from Ref. [26].

\begin{tabular}{lcccccc}
\hline \hline Nucl. & $G^{0 \nu}\left[\mathrm{yr}^{-1}\right]$ & $M^{\varepsilon}$ & $M^{\nu}$ & $f_{\text {nme }}$ & $T_{1 / 2}^{0 \nu-\exp }[y r] \operatorname{Ref}$. & $\left|\varepsilon_{e e}\right|$ \\
\hline${ }^{76} \mathrm{Ge}$ & $0.237 \times 10^{-14}$ & 5140 & 5.16 & $1.27 \times 10^{-3}$ & $>8.0 \times 10^{25}[27]$ & $<3.52 \times 10^{-10}$ \\
${ }^{82} \mathrm{Se}$ & $1.018 \times 10^{-14}$ & 4702 & 4.64 & $1.25 \times 10^{-3}$ & $>2.4 \times 10^{24}[28]$ & $<1.07 \times 10^{-9}$ \\
${ }^{100} \mathrm{Mo}$ & $1.595 \times 10^{-14}$ & 5751 & 5.40 & $1.19 \times 10^{-3}$ & $>1.1 \times 10^{24}[29]$ & $<1.03 \times 10^{-9}$ \\
${ }^{116} \mathrm{Cd}$ & $1.673 \times 10^{-14}$ & 3232 & 4.04 & $1.59 \times 10^{-3}$ & $>2.2 \times 10^{23}[30]$ & $<4.02 \times 10^{-9}$ \\
${ }^{130} \mathrm{Te}$ & $1.425 \times 10^{-14}$ & 4530 & 3.89 & $1.09 \times 10^{-3}$ & $>3.2 \times 10^{25}[31]$ & $<2.57 \times 10^{-10}$ \\
${ }^{136} \mathrm{Xe}$ & $1.462 \times 10^{-14}$ & 2530 & 2.18 & $1.09 \times 10^{-3}$ & $>1.07 \times 10^{26}[21]$ & $<2.49 \times 10^{-10}$ \\
\hline \hline
\end{tabular}

$$
a_{\nu} f_{\mathrm{nme}} \simeq-2.00 \times 10^{-7} .
$$

Recall that, the contributions to $0 \nu \beta \beta$-decay amplitude of the diagrams in Figs. 3(a) and 3(b) are proportional to the first and second terms in vertical brackets of Eq. (24), respectively. Thus, due to (28) the diagram Fig. 3(b) dominates in our scenario.

From the currently most stringent upper bound on the $0 \nu \beta \beta$-decay half-life obtained for ${ }^{136} \mathrm{Xe}$ by the KamLANDZen experiment [21]

$$
T_{1 / 2}^{0 \nu}>1.07 \times 10^{26} \mathrm{yr} \quad \text { at } 90 \% \text { C.L. }
$$

we find, using (24), an upper bound

$$
\left|\varepsilon_{e e}\right|<2.49 \times 10^{-10}
$$

Comparing the limit (30) for $\varepsilon_{e e}$ with the excluded regions in the first plot of Fig. 2, derived from the neutrinooscillation data, we conclude that the QCSS predicts the $\mathrm{NO}$ and a rather narrow interval of the lightest neutrino mass

$$
2.65 \mathrm{meV}<m_{0}=m_{1}<6.84 \mathrm{meV} .
$$

Using the $1 \sigma$ ranges of the neutrino-oscillation parameters $\sin ^{2} \theta_{i j}$ and $\Delta m_{i j}^{2}$ from Ref. [19], we derive according to Eq. (A3) the following ranges for the other two neutrino masses

$$
\begin{gathered}
9.0 \mathrm{meV}<m_{2}<11.2 \mathrm{meV}, \\
49.8 \mathrm{meV}<m_{3}<50.8 \mathrm{meV} .
\end{gathered}
$$

From the limits (31)-(32) we find the corresponding range for the cosmological neutrino parameter

$$
61.4 \mathrm{meV}<\Sigma=\sum_{i} m_{i}<68.8 \mathrm{meV} .
$$

This prediction of the QCSS is within the Planck limit (19). We also derive the QCSS range for the single-beta-decay parameter

$$
9.0 \mathrm{meV}<m_{\beta}=\sqrt{\sum_{i}\left|U_{e i}\right|^{2} m_{i}^{2}}<11.4 \mathrm{meV},
$$

which is beyond the reach of the current and near future tritium beta-decay experiments (for a recent review see, for instance, Refs. [32,33]).

At last, the limit (30) translated into the $0 \nu \beta \beta$-decay parameter gives the upper bound

$$
\left|m_{\beta \beta}\right|<2.33 \times 10^{-5} \mathrm{meV} .
$$

It is worth recalling that in the QCSS this parameter characterizes the subdominant contribution to the $0 \nu \beta \beta$ decay shown in Fig. 3(a), while the dominant one is given by the diagram Fig. 3(b).

\section{PARTICULAR REALIZATION OF QCSS MODEL}

There is one potential flaw in the model described above: the quark bilinear $\left(\overline{d_{R}} Q\right)$, being a $\mathcal{G}$-singlet, allows the $d$-quark tree-level Yukawa coupling shown in (8). It leads to a tree-level $d$-quark mass $m_{d}$ after the EWSB, which makes its the smallness rather weird. The common wisdom, allowing to avoid a fine-tuning, is to impose on theory an additional softly broken symmetry forbidding the tree-level Yukawa couplings of the light quarks, but allowing them at certain loop level (for a recent review see, for instance Ref. [1]). To this end we can extend the previously used group $\mathcal{G}$ to $\mathcal{G}^{\prime}=\mathcal{G} \times \mathcal{G}^{d}$ requiring that all the fields, except for $d$-quark, be neutral with respect to the subgroup $\mathcal{G}^{d}$. In this way we can forbid with the help of $\mathcal{G}^{d}$ the tree-level dquark Yukawa coupling. Once this symmetry is softly broken, the $d$-quark Yukawa can appear at loop level. In this case the d-quark mass could gain the necessary loop suppression. Let us give an example of such symmetry group $\mathcal{G}^{\prime}$ of our model

$$
\mathcal{G}^{\prime}=\mathbb{Z}_{4} \times \mathbb{Z}_{2} \stackrel{\text { soft }}{\rightarrow} \mathbb{Z}_{4}
$$

with the following $\mathbb{Z}_{4} \times \mathbb{Z}_{2}$-assignment of the fields 


$$
\begin{aligned}
& H \sim(1,1), \quad Q \sim(1,1), \quad u_{R} \sim(-1,1), \\
& d_{R} \sim(1,-1), \quad L \sim(i, 1), \quad e_{R} \sim(i,-1) .
\end{aligned}
$$

Here, we limit ourselves only to the first generation of the fermions. With this field assignment the Yukawa couplings of $u$ and $d$-quarks (8), the analogous electron coupling $H \bar{L} e_{R}$, as well as the operators $\mathcal{O}_{W}$ in Eq. (1), $\mathcal{O}_{7}^{d}$ in Eq. (3) and $\mathcal{O}_{6}^{u d}$ in Eq. (10) are all forbidden by $\mathbb{Z}_{4} \times \mathbb{Z}_{2}$ group. On the other hand, this group allows the operators $\mathcal{O}_{7}^{u}$ in Eq. (3) and $\mathcal{O}_{6}^{q q}$ in Eq. (10). Soft symmetry breaking (37) let the $d$-quark and electron Yukawa couplings arise at certain loop level. As a result these couplings acquire loop suppression factors necessary to make $m_{d}$ and $m_{e}$ smaller in comparison to the other SM fermions. The loop order depends on the concrete UV model. In principle, we can introduce an extra loop suppression to the electron Yukawa couplings in order to achieve $m_{d}>m_{e}$. This can be easily done by the extension of the scenario (37) to

$$
\mathcal{G}^{\prime \prime}=\mathbb{Z}_{4} \times \mathbb{Z}_{2} \times \mathbb{Z}_{2}^{e \text { soft }[1]} \mathbb{Z}_{4} \times \mathbb{Z}_{2}^{e} \stackrel{\text { soft[2] }}{\longrightarrow} \mathbb{Z}_{4}
$$

with all the field neutral with respect to $\mathbb{Z}_{2}^{e}$, but electron having $(-1)$ assignment to this subgroup. In this case the electron Yukawa appears at the second stage of the soft symmetry breaking chain and, therefore, can be realized at higher loop order than $d$-quark one. In this way the SM fermion mass hierarchy can be generated by sequential loop suppression [34] resulting from certain chain of soft symmetry breaking [7].

\section{CONCLUSION AND DISCUSSIONS}

We studied the quark condensate seesaw (QCSS) mechanism of generation of the Majorana neutrino mass matrix due to spontaneous breaking of chiral symmetry. The effect of the formation of chiral condensate is transmitted to the neutrino sector via the dimension-7 quark-lepton operators $\mathcal{O}_{7}^{u}$ in Eq (3). They can originate in low-energy limit from a certain class of UV models. We imposed on these models a symmetry, $\mathcal{G}$, forbidding the Weinberg operator $\mathcal{O}_{W}$ in Eq. (1) while allowing the operators $\mathcal{O}_{7}^{u}$. In this case the QCSS mechanism dominates over the ordinary tree-level Majorana neutrino mass generated by the electroweak symmetry breaking. The symmetry $\mathcal{G}$ inevitably forbids the $u$-quark Yukawa coupling making its mass equal to zero at a high-energy cutoff scale. We argued, following the existing literature, that $u$-quark receives a nonzero effective mass $m_{u}^{\text {eff }}$ from nonperturbative QCD effects at the scale $\Lambda_{\mathrm{QCD}} \sim 100 \mathrm{MeV}$. We commented that $m_{u}^{\text {eff }}$ generated in this way is compatible with the light hadron mass spectrum, but shows certain tension with the lattice simulations. A detailed study of this issue will be carried out elsewhere.

We discussed how in this scenario $d$-quark and electron can be made naturally lighter than other SM fermions.
We proposed to introduce a softly broken symmetry (37) or (39) forbidding the tree-level $d$-quark and electron Yukawa couplings, but unlocking them at some loop level. This mechanisms can bring into $d$-quark and electron masses, $m_{d}$ and $m_{e}$, loop-suppression factors necessary for making them naturally small. The order of the loop suppression depends on the particular UV model. We postpone the study of the possible UV completions of the QCSS scenario for the subsequent publication. We also noted that the $u$, $d$-quark masses always receive a contribution proportional to the chiral condensate via four-quark operators generated by nonperturbative QCD effects, which convert these quarks to the constituent ones.

We derived the predictions of the QCSS model for the LNV lepton-quark couplings (17). These couplings characterize the nonstandard neutrino-quark and charged leptonquark interactions arising from the operators in Eq. (3). They can be relevant for further studies of the phenomenological and astrophysical implications of the QCSS mechanism.

We analyzed the predictions of the QCSS model for neutrinoless double-beta decay. We calculated the corresponding nuclear matrix elements within the quasiparticle random phase approximation (QRPA) method with partial restoration of the isospin symmetry.

We commented about the role of the nuclear-matter effects for the neutrino mass mechanism in Fig. 3(a). We showed that the neutrino mass independent mechanism in Fig. 3(b) dominates in the QCSS scenario (9).

We found that the QCSS predicts the normal ordering (NO) of the neutrino-mass spectrum and rather narrow ranges (31)-(32) for the neutrino masses. This is in accord with the recent global analysis of the neutrino-oscillation data, which favors NO over IO at more than $3 \sigma$ [19]. We also derived predictions of the QCSS for some other observables (34)-(36).

\section{ACKNOWLEDGMENTS}

This work was supported by the Ministry of Education, Youth and Sports of the Czech Republic under the INAFYM Grant No. CZ.02.1.01/0.0/0.0/16_019/0000766 and the Grant of the Plenipotentiary Representative of the Czech Republic in JINR under Contract No. 202 from 24/ 03/2020, Fondo Nacional de Desarrollo Científico y Tecnológico (FONDECYT, Chile) No. 1190845 and Agencia Nacional de Investigación y Desarrollo (ANID, Chile) AFB180002, the RFBR Grant No. 18-02-00733 (Russia), and the VEGA Grant Agency of the Slovak Republic under Contract No. 1/0607/20.

\section{APPENDIX A: MAJORANA MASS MATRIX}

Diagonalizing the complex symmetric $3 \times 3$ Majorananeutrino mass matrix $m^{\nu}$ in Eqs. (4) and (6) $U^{\mathrm{T}} m_{\nu} U=$ $\operatorname{diag}\left(m_{1}, m_{2}, m_{3}\right)$ with a unitary lepton mixing matrix $U$, one gets the usual relation 


$$
\nu_{\alpha L}=\sum_{i} U_{\alpha i} \nu_{i L}
$$

between the neutrino mass eigenstates $\nu_{i}$ with masses $m_{i}$ and the flavor eigenstates $\nu_{\alpha}$. The matrix $U$ is known as the Pontecorvo-Maki-Nakagawa-Sakata (PMNS) matrix

$$
\begin{aligned}
U= & \left(\begin{array}{ccc}
1 & 0 & 0 \\
0 & c_{23} & s_{23} \\
0 & -s_{23} & c_{23}
\end{array}\right)\left(\begin{array}{ccc}
c_{13} & 0 & s_{13} e^{-i \delta} \\
0 & 1 & 0 \\
-s_{13} e^{i \delta} & 0 & c_{23}
\end{array}\right) \\
& \times\left(\begin{array}{ccc}
c_{12} & s_{12} & 0 \\
-s_{12} & c_{12} & 0 \\
0 & 0 & 1
\end{array}\right)\left(\begin{array}{ccc}
e^{i \alpha_{1}} & 0 & 0 \\
0 & e^{i \alpha_{2}} & 0 \\
0 & 0 & 1
\end{array}\right),
\end{aligned}
$$

parametrized in terms of the mixing angles $\theta_{12}, \theta_{13}, \theta_{23}$ $\left(s_{i j} \equiv \sin \theta_{i j}, c_{i j} \equiv \cos \theta_{i j}\right.$ ), Dirac phase $\delta$ and Majorana phases $\alpha_{1}, \alpha_{2}$ [35].

The neutrino masses $m_{i}(i=1,2,3)$ can be parametrized by the lightest-neutrino mass $m_{0}$ (which is unknown) and the mass-squared splittings $\Delta m_{i j}^{2}=m_{i}^{2}-m_{j}^{2}$ (known from the neutrino-oscillations experiments) for two types of the neutrino-mass ordering as

Normal ordering $(N O)$ with $m_{1}<m_{2} \ll m_{3}$ :

$$
\begin{aligned}
& m_{1}=m_{0}, \quad m_{2}=\sqrt{m_{0}^{2}+\Delta m_{21}^{2}}, \\
& m_{3}=\sqrt{m_{0}^{2}+\Delta m_{31}^{2}} .
\end{aligned}
$$

Inverted ordering $(I O)$ with $m_{3} \ll m_{1}<m_{2}$ :

$$
\begin{aligned}
& m_{1}=\sqrt{m_{0}^{2}-\Delta m_{31}^{2}}, \\
& m_{2}=\sqrt{m_{0}^{2}+\Delta m_{21}^{2}-\Delta m_{31}^{2}}, m_{3}=m_{0} .
\end{aligned}
$$

Elements $m_{\alpha \beta}^{\nu}=m_{\beta \alpha}^{\nu}$ of the Majorana mass matrix depend on the Dirac phase $\delta \in[0,2 \pi)$, Majorana phases $\alpha_{1}, \alpha_{2} \in[0, \pi)$, and neutrino masses $m_{i}$ determined by the lightest-neutrino mass $m_{0}$ and neutrino-mass ordering (NO or IO). Namely

$$
\begin{aligned}
m_{e e}^{\nu}= & c_{12}^{2} c_{13}^{2} e^{-i 2 \alpha_{1}} m_{1}+s_{12}^{2} c_{13}^{2} e^{-i 2 \alpha_{2}} m_{2}+s_{13}^{2} e^{i 2 \delta} m_{3}, \\
m_{e \mu}^{\nu}= & -c_{12} c_{13}\left(s_{12} c_{23}+c_{12} s_{13} s_{23} e^{-i \delta}\right) e^{-i 2 \alpha_{1}} m_{1} \\
& +s_{12} c_{13}\left(c_{12} c_{23}-s_{12} s_{13} s_{23} e^{-i \delta}\right) e^{-i 2 \alpha_{2}} m_{2} \\
& +s_{13} c_{13} s_{23} e^{i \delta} m_{3}, \\
m_{e \tau}^{\nu}= & c_{12} c_{13}\left(s_{12} s_{23}-c_{12} s_{13} c_{23} e^{-i \delta}\right) e^{-i 2 \alpha_{1}} m_{1} \\
& -s_{12} c_{13}\left(c_{12} s_{23}+s_{12} s_{13} c_{23} e^{-i \delta}\right) e^{-i 2 \alpha_{2}} m_{2} \\
& +s_{13} c_{13} c_{23} e^{i \delta} m_{3},
\end{aligned}
$$

$$
\begin{aligned}
m_{\mu \mu}^{\nu}= & \left(s_{12} c_{23}+c_{12} s_{13} s_{23} e^{-i \delta}\right)^{2} e^{-i 2 \alpha_{1}} m_{1} \\
& +\left(c_{12} c_{23}-s_{12} s_{13} s_{23} e^{-i \delta}\right)^{2} e^{-i 2 \alpha_{2}} m_{2}+c_{13}^{2} s_{23}^{2} m_{3},
\end{aligned}
$$

$$
\begin{aligned}
m_{\mu \tau}^{\nu}= & -\left(s_{12} s_{23}-c_{12} s_{13} c_{23} e^{-i \delta}\right) \\
& \times\left(s_{12} c_{23}+c_{12} s_{13} s_{23} e^{-i \delta}\right) e^{-i 2 \alpha_{1}} m_{1} \\
& -\left(c_{12} s_{23}+s_{12} s_{13} c_{23} e^{-i \delta}\right) \\
& \times\left(c_{12} c_{23}-s_{12} s_{13} s_{23} e^{-i \delta}\right) e^{-i 2 \alpha_{2}} m_{2}+c_{13}^{2} s_{23} c_{23} m_{3},
\end{aligned}
$$

$$
\begin{aligned}
m_{\tau \tau}^{\nu}= & \left(s_{12} s_{23}-c_{12} s_{13} c_{23} e^{-i \delta}\right)^{2} e^{-i 2 \alpha_{1}} m_{1} \\
& +\left(c_{12} s_{23}+s_{12} s_{13} c_{23} e^{-i \delta}\right)^{2} e^{-i 2 \alpha_{2}} m_{2}+c_{13}^{2} c_{23}^{2} m_{3} .
\end{aligned}
$$

We employ these relations for the analysis of the nonstandard neutrino-quark couplings (7) and (17), which is done in Sec. III. In this analysis, we use the neutrinooscillation data from Ref. [19].

\section{APPENDIX B: QUARK CONDENSATE}

Chiral symmetry is approximate invariance of the QCD Lagrangian under the global $\mathrm{SU}(3)_{L} \times \mathrm{SU}(3)_{R}$ transformations in the space of the lightest quark flavors $q=u, d$, $s$. Below the chiral scale $4 \pi f_{\pi} \sim 1 \mathrm{GeV}$, this symmetry is spontaneously broken by the light-quark condensates $\langle 0|\bar{q} q| 0\rangle \equiv\langle\bar{q} q\rangle \neq 0$ in the QCD ground state (vacuum) $|0\rangle$. The corresponding Goldstone bosons form the octet of light mesons. Their nonzero masses originate from the explicit breaking of the chiral symmetry by the light-quark current mass terms in the QCD Lagrangian/Hamiltonian:

$$
\begin{aligned}
\mathcal{H}_{\mathrm{m}}= & m_{u} \bar{u} u+m_{d} \bar{d} d+m_{s} \bar{s} s \\
= & \frac{1}{2}\left(m_{u}+m_{d}\right)(\bar{u} u+\bar{d} d) \\
& +\frac{1}{2}\left(m_{u}-m_{d}\right)(\bar{u} u-\bar{d} d)+m_{s} \bar{s} s \\
= & 2 m_{q} \bar{q} q+\ldots,
\end{aligned}
$$

where we separated the isospin-singlet and isospin-triplet quark combinations. In what follows, we retain the singlet and consider only the lightest $u$ and $d$ quarks, denoting $m_{q}=\frac{1}{2}\left(m_{u}+m_{d}\right)$ and $\bar{q} q=\frac{1}{2}(\bar{u} u+\bar{d} d)$. Note that in our scenario the mass $m_{u}$ of u-quark, explicitly breaking chiral symmetry, originates not from the electroweak symmetry breaking, which is the case for all the other current quark masses, but from nonperturbative QCD. Thus, throughout this section, it is implied that $m_{u}=m_{u}^{C h S B}$, as defined in Eq. (14). 
Here, we will examine the effect of nuclear environment on the formation of light quark condensate. Following Ref. [36], we use the Hellmann-Feynman theorem, allowing one to analyze the condensates in a modelindependent way to the first order in nucleon density. The Hellmann-Feynman theorem states

$$
\left\langle\psi(\lambda)\left|\frac{\mathrm{d}}{\mathrm{d} \lambda} H(\lambda)\right| \psi(\lambda)\right\rangle=\frac{\mathrm{d}}{\mathrm{d} \lambda} E(\lambda),
$$

where $|\psi(\lambda)\rangle$ and $E(\lambda)$ are, respectively, the normalized energy eigenstates and eigenvalues of the Hamiltonian $H(\lambda)$ with explicit dependence on the parameter $\lambda$. Choosing $\lambda=m_{q}$ and $H=\int \mathrm{d}^{3} \vec{x} \mathcal{H}_{\mathrm{m}}$ we get

$$
2 m_{q}\left\langle\psi\left(m_{q}\right)\left|\int \mathrm{d}^{3} \vec{x} \bar{q} q\right| \psi\left(m_{q}\right)\right\rangle=m_{q} \frac{\mathrm{d} E\left(m_{q}\right)}{\mathrm{d} m_{q}},
$$

where both parts of this equation are multiplied by $m_{q}$ to ensure renormalization-group invariance of this relation [37]. Let us consider two cases $\left.\left|\psi\left(m_{q}\right)=\right| 0\right\rangle,\left|\rho_{N}\right\rangle$, where $|0\rangle$ is the QCD vacuum and $\left|\rho_{N}\right\rangle$ refers to the ground state of (uniform) nuclear matter at rest with nucleon density $\rho_{N}$. Writing Eq. (B3) for these two cases, we subtract one from the other and obtain

$$
2 m_{q}\left(\left\langle\rho_{N}|\bar{q} q| \rho_{N}\right\rangle-\langle 0|\bar{q} q| 0\rangle\right)=m_{q} \frac{\mathrm{d} \mathcal{E}_{N}}{\mathrm{~d} m_{q}},
$$

where $\mathcal{E}_{N}$ is the energy density of nuclear matter. Provided the kinetic and potential energy of nucleons are known to be small, one has

$$
\mathcal{E}_{N}=m_{N} \rho_{N}
$$

On the other hand, there is a current-algebra relation [38]

$$
\sigma_{N}=m_{q} \frac{\mathrm{d} m_{N}}{\mathrm{~d} m_{q}}
$$

where $\sigma_{N}$ is the pion-nucleon sigma term measuring the nucleon-mass $m_{N}$ shift from the chiral limit $m_{u, d} \rightarrow 0$. Then, using Eqs. (B4), (B5), and (B6), one finds a modelindependent relation [36]

$$
\frac{\langle\bar{q} q\rangle_{N}}{\langle\bar{q} q\rangle}=1+\frac{\sigma \rho_{N}}{2 m_{q}\langle\bar{q} q\rangle}=1-\frac{\rho \sigma_{N}}{f_{\pi}^{2} m_{\pi}^{2}},
$$

characterizing the amount of chiral-symmetry restoration in dense medium. Here, we denoted $\langle\bar{q} q\rangle_{N} \equiv\left\langle\rho_{N}|\bar{q} q| \rho_{N}\right\rangle$ and $\langle\bar{q} q\rangle \equiv\langle 0|\bar{q} q| 0\rangle$. The Gell-Mann-Oakes-Renner relation [39]

$$
2 m_{q}\langle\bar{q} q\rangle=-f_{\pi}^{2} m_{\pi}^{2}
$$

has been used to derive Eq. (B7). In order to estimate the nuclear-matter effect on the quark condensate on the basis of Eq. (B7), we adopt the usual value for the nucleon density $\rho=\rho_{p}+\rho_{n}=0.17 \mathrm{fm}^{-3}$, the recent large value of $\sigma=64 \mathrm{MeV}$ from a partial-wave analysis of the $\pi-N$ scattering [40], $f_{\pi}=92 \mathrm{MeV}$ and the charged-pion mass $m_{\pi}=140 \mathrm{MeV}$. Then, Eq. (B7) yields

$$
\langle\bar{q} q\rangle_{N} \approx 0.5\langle\bar{q} q\rangle,
$$

demonstrating a substantial suppression of the quark condensate in the nuclear matter. The value $\langle\bar{q} q\rangle_{N}$ can be interpreted as the sum of scalar densities of the $u$ (or $d$ ) quarks in vacuum and inside nucleons. The nucleon component of $\langle\bar{q} q\rangle_{N}$ is estimated in Ref. [23] to be $\approx(100 \mathrm{MeV})^{3}$. The sign of the nucleon component is opposite to the sign of the vacuum component; the latter is also numerically higher.

\section{APPENDIX C: THE 0 $\nu \beta \beta$-DECAY RATE AND NUCLEAR MATRIX ELEMENTS}

The $\beta$-decay Hamiltonian contains standard model and nonstandard neutrino interactions

$$
\begin{aligned}
H^{\beta}= & \frac{G_{\beta}}{\sqrt{2}} \bar{e} \gamma^{\rho}\left(1-\gamma_{5}\right) \nu_{e} \bar{u} \gamma_{\rho}\left(1-\gamma_{5}\right) d \\
& +\frac{G_{\beta}}{\sqrt{2}} \frac{\varepsilon_{e e}}{4} \bar{e}\left(1+\gamma_{5}\right) \nu_{e}^{C} \bar{u}\left(1-\gamma_{5}\right) d+\text { H.c. }
\end{aligned}
$$

Here, $G_{\beta}=G_{F} \cos \theta_{C}$, where $\cos \theta_{C}$ is the Cabbibo angle.

By the higher order perturbation of strong and electromagnetic interactions quark currents are converted into the ones involving nucleons. We have

$$
\begin{aligned}
& \left\langle n\left(p^{\prime}\right)\left|\bar{d} \gamma^{\alpha}\left(1-\gamma_{5}\right) u\right| p(p)\right\rangle \\
& =\bar{n}\left(p^{\prime}\right)\left[g_{V}\left(q^{2}\right) \gamma^{\alpha}\right. \\
& \left.\quad+i g_{M}\left(q^{2}\right) \frac{\sigma^{\alpha \beta}}{2 m_{p}} q_{\beta}-g_{A}\left(q^{2}\right) \gamma^{\alpha} \gamma_{5}-g_{P}\left(q^{2}\right) q^{\alpha} \gamma_{5}\right] p(p), \\
& \left\langle n\left(p^{\prime}\right)\left|\bar{d}\left(1-\gamma_{5}\right) u\right| p(p)\right\rangle \\
& \quad=\bar{n}\left(p^{\prime}\right)\left[g_{S}\left(q^{2}\right)-g_{P S}\left(q^{2}\right) \gamma_{5}\right] p(p), \quad \text { (C2) }
\end{aligned}
$$

where $m_{p}$ is the nucleon mass, $q_{\mu}=\left(p^{\prime}-p\right)_{\mu}$ is the momentum transfer and $p^{\prime}$ and $p$ are the four momenta of neutron and proton, respectively. For the nucleon form factors $g_{V}\left(q^{2}\right), g_{M}\left(q^{2}\right), g_{A}\left(q^{2}\right)$ and $g_{P}\left(q^{2}\right)$ we use parametrization as follows [41]: 


$$
\begin{aligned}
\frac{g_{V, M, S}\left(q^{2}\right)}{g_{V, M, S}} & =\left(1+\frac{q^{2}}{m_{V}^{2}}\right)^{-2}, \quad \frac{g_{A}\left(q^{2}\right)}{g_{A}}=\left(1+\frac{q^{2}}{m_{A}^{2}}\right)^{-2}, \\
\frac{g_{P S}\left(q^{2}\right)}{g_{P S}} & =\left(1+\frac{q^{2}}{m_{A}^{2}}\right)^{-2}\left(1+\frac{q^{2}}{m_{\pi}^{2}}\right)^{-1} .
\end{aligned}
$$

Here, $m_{\pi}$ is the mass of pion, $q^{2}=\mathbf{q} \cdot \mathbf{q}$ (a small energy transfer in the nucleon vertex can be neglected), $m_{V}=$ $0.84 \mathrm{GeV}$ and $m_{A}=1.09 \mathrm{GeV}$ and the renormalization constants: $g_{V}=1, g_{A}=1.269, g_{M}=\left(\mu_{p}-\mu_{n}\right)=3.70$, $g_{S}=1.0[42,43]$ and $g_{P S}=349$ [43]. The induced pseudoscalar coupling is given by the PCAC relation

$$
g_{P}\left(q^{2}\right)=\frac{2 m_{p}}{q^{2}+m_{\pi}^{2}} g_{A}\left(q^{2}\right) .
$$

To obtain nuclear matrix elements of interest, nonrelativistic expansion of nucleon matrix elements in Eq. (C2) have to be performed. For nuclear currents we get

$J_{V-A}^{\mu^{\dagger}}(\mathbf{x})=\sum_{n=1}^{A} \tau_{n}^{+}\left[g^{\mu 0} J_{V-A}^{0}(\mathbf{q})+g^{\mu k} J_{V-A}^{k}(\mathbf{q})\right] \delta\left(\mathbf{x}-\mathbf{r}_{n}\right)$,

$J_{S-P}^{\dagger}(\mathbf{x})=\sum_{n=1}^{A} \tau_{n}^{+} J_{S P}(\mathbf{q}) \delta\left(\mathbf{x}-\mathbf{r}_{n}\right)$

with $k=1,2,3$ and

$$
\begin{aligned}
& J_{V-A}^{0}(\mathbf{q})=g_{V}\left(q^{2}\right) \\
& \mathbf{J}_{V-A}(\mathbf{q})=g_{M}\left(q^{2}\right) i \frac{\boldsymbol{\sigma} \times \mathbf{q}}{2 m_{p}}-g_{A}\left(q^{2}\right) \boldsymbol{\sigma}+g_{P}\left(q^{2}\right) \frac{\mathbf{q} \boldsymbol{\sigma} \cdot \mathbf{q}}{2 m_{p}} \\
& J_{S-P}(\mathbf{q})=g_{S}\left(q^{2}\right)-g_{P S}\left(q^{2}\right) \frac{\boldsymbol{\sigma} \cdot \mathbf{q}}{2 m_{p}}
\end{aligned}
$$

where $\mathbf{r}_{n}$ is the coordinate of the $n$th nucleon.

The nucleon recoil terms associated with the initial and final vertices in the $0 \nu \beta \beta$-decay transition contain the nucleon recoil momenta $\mathbf{q}_{m}$ and $\mathbf{q}_{n}$, respectively. They are opposite in direction and their absolute values $q_{m}=$ $\left|\mathbf{q}_{m}\right|$ and $q_{m}=\left|\mathbf{q}_{n}\right|$ are roughly equal to the absolute value of neutrino momentum $p=|\mathbf{p}|$. We have [44]

$$
\mathbf{q}_{m} \simeq-\mathbf{q}_{n} \simeq \mathbf{p} .
$$

In the amplitude only linear term in $\varepsilon_{e e}$ is considered. The main contribution to corresponding nuclear matrix element for ground state to ground state $0^{+} \rightarrow 0^{+}$transition is given by combinations of $g_{P S}$ term with $g_{A}$ and $g_{P}$ terms of nucleon currents and the spatial component of neutrino propagator proportional to neutrino momentum $\mathbf{p}$.

For the inverse $0 \nu \beta \beta$-decay half-life we obtain

$$
\frac{1}{T_{1 / 2}}=G^{0 \nu}\left|\varepsilon_{e e} g_{A} M^{\varepsilon}+\frac{m_{\beta \beta}}{m_{e}} g_{A}^{2} M^{\nu}\right|^{2}
$$

Here, $G^{0 \nu}$ is the known phase-space factor. Nuclear matrix elements $M^{\nu}$ and $M^{\varepsilon}$, depend on the nuclear structure of the particular isotopes $(A, Z),(A, Z+1)$ and $(A, Z+2)$ under study. The explicit form of $M^{\nu}$ can be found, e.g., in [25,45] and $M^{\varepsilon}$ is presented in a similar way here.

The nuclear matrix element $\mathcal{M}^{\varepsilon}$ consists of the GamowTeller (GT) and tensor (T) parts

$$
\mathcal{M}^{\varepsilon}=\mathcal{M}_{\mathrm{GT}}^{\varepsilon}+\mathcal{M}_{\mathrm{T}}^{\varepsilon} .
$$

In the QRPA method, $\mathcal{M}^{\nu, \varepsilon}$ is written via sums over the virtual intermediate states labeled by their angular momenta and parities $J^{\pi}$ and indices $k_{i}$ and $k_{f}[25,45]$

$$
\begin{aligned}
& \mathcal{M}_{K}=\sum_{J^{\pi}, k_{i}, k_{f}, \mathcal{J}} \sum_{p n p^{\prime} n^{\prime}}(-1)^{j_{n}+j_{p^{\prime}}+J+\mathcal{J}} \sqrt{2 \mathcal{J}+1} \\
& \times\left\{\begin{array}{lll}
j_{p} & j_{n} & J \\
j_{n^{\prime}} & j_{p^{\prime}} & \mathcal{J}
\end{array}\right\}\left\langle p(1), p^{\prime}(2) ; \mathcal{J}\left\|\mathcal{O}_{K}\right\| n(1), n^{\prime}(2) ; \mathcal{J}\right\rangle \\
& \times\left\langle 0_{f}^{+}\left\|\left[c_{p^{\prime}}^{\dagger} \tilde{c}_{n^{\prime}}\right]_{J}\right\| J^{\pi} k_{f}\right\rangle\left\langle J^{\pi} k_{f} \mid J^{\pi} k_{i}\right\rangle\left\langle J^{\pi} k_{f} i\left\|\left[c_{p}^{\dagger} \tilde{c}_{n}\right]_{J}\right\| 0_{i}^{+}\right\rangle .
\end{aligned}
$$

The reduced matrix elements of the one-body operators $c_{p}^{\dagger} \tilde{c}_{n}\left(\tilde{c}_{n}\right.$ denotes the time-reversed state) in the Eq. (C10) depend on the BCS coefficients $u_{i}, v_{j}$ and on the QRPA vectors $X, Y$ [25].

The two-body operators $O_{K}^{\varepsilon}$ in (C10), where $K=\mathrm{GT}$, T (Tensor), contain neutrino potentials, spin and isospin operators, and RPA energies $E_{J^{n}}^{k_{i}, k_{f}}$ :

$$
\begin{aligned}
O_{\mathrm{GT}}^{\varepsilon}\left(r_{12}, E_{J^{n}}^{k}\right) & =\tau^{+}(1) \tau^{+}(2) H_{\mathrm{GT}}^{\varepsilon}\left(r_{12}, E_{J^{n}}^{k}\right) \sigma_{12}, \\
O_{\mathrm{T}}^{\varepsilon}\left(r_{12}, E_{J^{n}}^{k}\right) & =\tau^{+}(1) \tau^{+}(2) H_{\mathrm{T}}^{\varepsilon}\left(r_{12}, E_{J^{n}}^{k}\right) S_{12}
\end{aligned}
$$

with

$$
\begin{aligned}
\mathbf{r}_{12} & =\mathbf{r}_{\mathbf{1}}-\mathbf{r}_{\mathbf{2}}, r_{12} \equiv\left|\mathbf{r}_{\mathbf{1 2}}\right|, \quad \hat{\mathbf{r}}_{\mathbf{1 2}} \equiv \frac{\mathbf{r}_{\mathbf{1 2}}}{r_{12}}, \\
\sigma_{12} & =\boldsymbol{\sigma}_{\mathbf{1}} \cdot \boldsymbol{\sigma}_{\mathbf{2}}, \\
S_{12} & =3\left(\boldsymbol{\sigma}_{\mathbf{1}} \cdot \hat{\mathbf{r}}_{\mathbf{1 2}}\right)\left(\boldsymbol{\sigma}_{\mathbf{2}} \cdot \hat{\mathbf{r}}_{\mathbf{1 2}}\right)-\sigma_{12} .
\end{aligned}
$$

Here, $\mathbf{r}_{\mathbf{1}}$ and $\mathbf{r}_{\mathbf{2}}$ are the coordinates nucleons undergoing the beta decay.

The neutrino potentials are

$H_{K}^{\varepsilon}\left(r_{12}, E_{J^{\pi}}^{k}\right)=\frac{2}{\pi} R \int_{0}^{\infty} f_{K}\left(p r_{12}\right) \frac{h^{\varepsilon}\left(p^{2}\right) p \mathrm{~d} p}{p+E_{J^{\pi}}^{k}-\frac{1}{2}\left(E_{i}+E_{f}\right)}$,

where $f_{\mathrm{F}, \mathrm{GT}}\left(q r_{12}\right)=j_{0}\left(q r_{12}\right)$ and $f_{\mathrm{T}}\left(q r_{12}\right)=-j_{2}\left(q r_{12}\right)$ are the spherical Bessel functions. The potentials (C13) depend explicitly - though rather weakly — on the energies $E_{J^{n}}^{k}$ of the virtual intermediate states. The functions $h\left(p^{2}\right)$ in Eq. (C13) is defined as 
$h^{\varepsilon}\left(p^{2}\right)=\frac{1}{12} \frac{F_{P}^{(3)}\left(p^{2}\right) g_{A}\left(p^{2}\right)}{g_{A}}\left(1-\frac{p^{2}}{p^{2}+m_{\pi}^{2}}\right) \frac{p^{2}}{m_{p} m_{e}}$.

In Table I, we show nuclear matrix elements $M^{\varepsilon}, M^{\nu}$ and their ratio $f_{\mathrm{NME}}$ calculated within the QRPA method with partial restoration of the isospin symmetry. Details of the calculation are given in [25]. By glancing at Table I we see that $M^{\varepsilon}$ is by about factor 200 larger when compared with $M^{\nu}$, what is a result of the additional factor $p /\left(2 m_{e}\right)$ in the neutrino exchange potential [see Eq. (C13)]. It is worth noting that within considered approximations $M^{\varepsilon}$ does not depend on axial-vector coupling constant $g_{A}$. The most stringent current experimental limits on the $0 \nu \beta \beta$-decay half-life and corresponding upper bounds on the absolute value of $\varepsilon_{e e}$ are presented in Table I as well.
[1] Y. Cai, J. Herrero-García, M. A. Schmidt, A. Vicente, and R. R. Volkas, Front. Phys. 5, 63 (2017).

[2] E. Ma and O. Popov, Phys. Lett. B 764, 142 (2017).

[3] C.-Y. Yao and G.-J. Ding, Phys. Rev. D 96, 095004 (2017); 98, 039901(E) (2018).

[4] S. Centelles Chuliá, R. Srivastava, and J. W. F. Valle, Phys. Rev. D 98, 035009 (2018).

[5] S. Centelles Chuliá, R. Cepedello, E. Peinado, and R. Srivastava, J. High Energy Phys. 10 (2019) 093.

[6] C. Arbeláez, A. E. Cárcamo, R. Cepedello, M. Hirsch, and S. Kovalenko, Phys. Rev. D 100, 115021 (2019).

[7] C. Arbeláez, A. E. Cárcamo Hernández, R. Cepedello, S. Kovalenko, and I. Schmidt, J. High Energy Phys. 06 (2020) 043.

[8] K. S. Babu, P. S. Bhupal Dev, S. Jana, and A. Thapa, J. High Energy Phys. 03 (2020) 006.

[9] H. Päs, M. Hirsch, H. Klapdor-Kleingrothaus, and S. Kovalenko, Phys. Lett. B 453, 194 (1999).

[10] F. F. Deppisch, M. Hirsch, and H. Päs, J. Phys. G 39, 124007 (2012).

[11] C. Arbeláez, M. González, M. Hirsch, and S. Kovalenko, Phys. Rev. D 94, 096014 (2016); 97, 099904(E) (2018).

[12] V. Cirigliano, W. Dekens, J. de Vries, M. Graesser, and E. Mereghetti, J. High Energy Phys. 12 (2017) 082.

[13] S. D. Thomas and R.-M. Xu, Phys. Lett. B 284, 341 (1992).

[14] C. McNeile, A. Bazavov, C. T. H. Davies, R. J. Dowdall, K. Hornbostel, G. P. Lepage, and H. D. Trottier, Phys. Rev. D 87, 034503 (2013).

[15] C. Allton et al. (RBC-UKQCD Collaboration), Phys. Rev. D 78, 114509 (2008).

[16] N. Kitazawa and Y. Sakai, Int. J. Mod. Phys. A 33, 1850017 (2018).

[17] H. Davoudiasl and L. L. Everett, Phys. Lett. B 634, 55 (2006).

[18] We are thankful to Martin Hirsch for drawing our attention to this fact.

[19] P. F. de Salas, D. V. Forero, C. A. Ternes, M. Tórtola, and J. W. F. Valle, Phys. Lett. B 782, 633 (2018).

[20] N. Aghanim et al. (Planck Collaboration), Astron. Astrophys. 641, A6 (2020).

[21] A. Gando et al. (KamLAND-Zen Collaboration), Phys. Rev. Lett. 117, 082503 (2016).

[22] S. Vagnozzi, E. Giusarma, O. Mena, K. Freese, M. Gerbino, S. Ho, and M. Lattanzi, Phys. Rev. D 96, 123503 (2017).

[23] S. Kovalenko, M. I. Krivoruchenko, and F. Šimkovic, Phys. Rev. Lett. 112, 142503 (2014).
[24] J. C. Helo, S. Kovalenko, and I. Schmidt, Nucl. Phys. B853, 80 (2011).

[25] F. Šimkovic, V. Rodin, A. Faessler, and P. Vogel, Phys. Rev. C 87, 045501 (2013).

[26] D. Štefánik, R. Dvornický, F. Šimkovic, and P. Vogel, Phys. Rev. C 92, 055502 (2015).

[27] M. Agostini et al. (GERDA Collaboration), Phys. Rev. Lett. 120, 132503 (2018).

[28] O. Azzolini et al. (CUPID-0 Collaboration), Phys. Rev. Lett. 120, 232502 (2018).

[29] R. Arnold et al. (NEMO-3 Collaboration), Phys. Rev. D 92, 072011 (2015).

[30] V. I. Tretyak et al. (Aurora Collaboration), AIP Conf. Proc. 81, 020029 (2019).

[31] D. Q. Adams et al. (CUORE Collaboration), Phys. Rev. Lett. 124, 122501 (2020).

[32] G. Drexlin, V. Hannen, S. Mertens, and C. Weinheimer, Adv. High Energy Phys. 2013, 293986 (2013).

[33] M. Aker et al. (KATRIN Collaboration), Phys. Rev. Lett. 123, 221802 (2019).

[34] A. E. Cárcamo Hernández, S. Kovalenko, and I. Schmidt, J. High Energy Phys. 02 (2017) 125.

[35] F. Capozzi, E. Lisi, A. Marrone, D. Montanino, and A. Palazzo, Nucl. Phys. B908, 218 (2016).

[36] T. D. Cohen, R. J. Furnstahl, and D. K. Griegel, Phys. Rev. C 45, 1881 (1992).

[37] R. Tarrach, Nucl. Phys. B196, 45 (1982).

[38] R. L. Jaffe and C. L. Korpa, Comments Nucl. Part. Phys. 17, 163 (1987).

[39] M. Gell-Mann, R. J. Oakes, and B. Renner, Phys. Rev. 175, 2195 (1968).

[40] M. M. Pavan, I. I. Strakovsky, R. L. Workman, and R. A. Arndt, PiN Newslett. 16, 110 (2002).

[41] L. Graf, F. F. Deppisch, F. Iachello, and J. Kotila, Phys. Rev. D 98, 095023 (2018).

[42] R. Gupta, Y. Jang, B. Yoon, H. Lin, V. Cirigliano, and T. Bhattacharya, Phys. Rev. D 98, 034503 (2018).

[43] M. M. González-Alonso and J. Martin Camalich, Phys. Rev. Lett. 112, 042501 (2014).

[44] M. Doi, T. Kotani, and E. Takasugi, Prog. Theor. Phys. Suppl. 83, 1 (1985).

[45] F. Šimkovic, A. Faessler, V. Rodin, P. Vogel, and J. Engel, Phys. Rev. C 77, 045503 (2008). 